\title{
Performance Output Tracking for a One-Dimensional Wave-Heat Cascade System with Unmatched Disturbance
}

\author{
Yulong Liu \\ School of Mathematical Sciences, Shanxi University, Taiyuan, Shanxi, 030006, China \\ Correspondence should be addressed to Yulong Liu; liuylmath@139.com
}

Received 27 January 2019; Revised 18 March 2019; Accepted 27 March 2019; Published 15 April 2019

Academic Editor: Átila Bueno

Copyright ( 2019 Yulong Liu. This is an open access article distributed under the Creative Commons Attribution License, which permits unrestricted use, distribution, and reproduction in any medium, provided the original work is properly cited.

The objective of this paper is to solve the performance output regulation problem for a wave-heat cascade system with unmatched disturbance. Applying the series expansion, the auxiliary trajectory for the cascade system is constructed and the unmatched disturbance is rejected. Meanwhile, the controller and observer only based on error signal are designed, and the performance output regulation problem is solved. Under the control feedback, the performance output can track the reference signal, and the regulation error goes to zero asymptotically. Finally, some numerical simulations are presented for illustration.

\section{Introduction}

In recent years, regulating the output of a given distributed parameter system is one of the central problems in control theory. The objective of the output regulation is to construct a controller such that the performance output of the given plant can track a reference signal. Comparing with the finitedimensional system, there exist various so-called noncollocated problems in distributed parameter system. The system we consider is described by the one-dimensional wave-heat cascade system as follows:

$$
\begin{aligned}
\dot{v}(t) & =G v(t), \quad t \geq 0, \\
u_{t t}(x, t) & =u_{x x}(x, t), \quad x \in(0,1), \quad t>0, \\
u_{x}(0, t) & =c_{1} z(0, t)+c_{2} u(0, t), \quad t \geq 0, \\
u_{x}(1, t) & =-c_{0} u_{t}(1, t), \quad t \geq 0, \\
z_{t}(x, t) & =z_{x x}(x, t), \quad x \in(0,1), \quad t>0, \\
z_{x}(0, t) & =d(t), \quad t \geq 0, \\
z(1, t) & =U(t), \quad t \geq 0, \\
u(x, 0) & =u_{0}(x), \quad x \in[0,1], \\
u_{t}(x, 0) & =u_{1}(x), \quad x \in[0,1],
\end{aligned}
$$

$$
\begin{gathered}
z(x, 0)=z_{0}(x), \quad x \in[0,1], \\
Y_{\text {out }}=u(1, t), \quad t \geq 0,
\end{gathered}
$$

where $u_{0}, u_{1}$, and $z_{0}$ are the initial state, $Y_{\text {out }}$ is the performance output, $d(t)$ is the disturbance, and $U:[0, \infty) \longrightarrow \mathbb{R}$ is the control input. Considering the control plant (1) in the state space $\mathscr{H}=\mathbb{C}^{n} \times H^{1}(0,1) \times L^{2}(0,1)$, we are going to design a control law so that the performance output $Y_{\text {out }}$ tracks the given reference signal $Y_{r e f}$ in the presence of the external disturbance $d$.

Recently, the performance output tracking for a wave equation with harmonic disturbance is considered in [1] where the control and disturbance are unmatched. In [2], the same problem is also studied for a wave with a general boundary disturbance by the disturbance treatment technique which is firstly proposed in [3]. These results were extended to regulate the output of a Schrödinger equation in $[4,5]$. However, the literature mentioned above is considered in a relative easy situation where the performance output is always collocated to the control actuation. Therefore, one of the objectives of this paper is to deal with the problem that the performance output is not collocated to the control actuation. The latest progress of this problem is considered in [6-9]. They use the method of backstepping, servo system, and the adaptive control approach to solve 
the performance output regulation problem in noncollocated case. The proposed method design can also be extended to the more general control system. Meanwhile, $[10,11]$ construct various controllers to solve the robust output regulation of distribution parameter systems by internal model principle. Different from the methods mentioned above, in this paper, a novel auxiliary trajectory and servomechanism is designed to cope with the noncollocated problem for system (1).

In addition, the problem of the output regulation for cascaded system is seldom considered. Concerning the cascaded system, a lot of results have been obtained only for stabilization problem [12-15]. In view of this reason, another objective of this paper is to solve the problem of the output regulation for the cascaded system (1) by a novel method of auxiliary trajectory and servomechanism.

For the disturbance and the reference signal, in system (1), both $d(t)$ and $Y_{\text {ref }}$ are supposed to be the harmonic signals of the following form:

$$
\begin{gathered}
d(t)=\sum_{j=0}^{m} A_{j} \sin \omega_{j} t+B_{j} \cos \omega_{j} t, \quad m \in \mathbb{N}, \\
Y_{r e f}(t)=\sum_{k=0}^{\widetilde{m}} \widetilde{A}_{k} \sin \widetilde{\omega}_{k} t+\widetilde{B}_{k} \cos \widetilde{\omega}_{k} t, \quad \widetilde{m} \in \mathbb{N},
\end{gathered}
$$

where $\widetilde{A}_{k}, \widetilde{B}_{k}, A_{j}, B_{j}$ are unknown amplitudes and $\omega_{j}, \widetilde{\omega}_{k}$ are known frequencies, $j=1,2, \ldots, m, k=1,2, \ldots, \widetilde{m}$. Ву а simple computation, both of them can be rewritten as an output of the following exosystem:

$$
\begin{aligned}
& \dot{v}(t)=G v(t), \quad t \geq 0, \\
& Y_{r e f}=F v(t), \quad t \geq 0, \\
& d(t)=Q v(t), \quad t \geq 0, \\
& v(0)=v_{0},
\end{aligned}
$$

where $G \in \mathbb{R}^{n \times n}$ is the system matrix; $F$ and $Q$ are known $n$-dimensional row vectors; and the initial state $v_{0}$ depends on the amplitudes $\widetilde{A}_{k}, \widetilde{B}_{k}, A_{j}, B_{j}$ and hence is unknown. Throughout this paper, we always assume that $G$ is invertible and diagonalizable with $\sigma(G)=\left\{\lambda_{i}\right\}_{i=1}^{n} \subset i \mathbb{R} /\{0\}$ and $\operatorname{Re}\langle G x, x\rangle_{\mathbb{C}^{n}}=0, \forall x \in \mathbb{C}^{n}$. Under this assumption, the general harmonic signal can be written as an output of the exosystem (3). With this assumption, the mathematical foundations of the output regulation problem in question can be found in $[16,17]$.

Now, we need to design a controller such that the regulation error $Y_{e}=Y_{r e f}-Y_{o u t}$ (the only measurement for controller design) satisfies

$$
\lim _{t \longrightarrow \infty} Y_{e}(t)=\lim _{t \longrightarrow \infty}\left[Y_{\text {ref }}(t)-Y_{\text {out }}(t)\right]=0 .
$$

We proceed as follows. In Section 2, the servomechanism will be designed by the method of auxiliary trajectory and the negative impacts of the unmatched disturbance will be canceled. In Section 3, the controller will be designed and the performance output regulation problem will be solved under the control feedback. In Section 4, we design an observer based on error signal and prove the convergence of the observer by the Lyapunov functional method. In Section 5, the uniform boundedness of the loop system will be presented and proved. Section 6 presents some numerical simulations to illustrate the effectiveness of the control law, followed by concluding remarks in Section 7.

\section{Trajectory Planning for the Disturbance}

In this section, we are going to cancel the negative impacts of the disturbance by trajectory planning. We first consider the unmatched disturbance $Q v(t)$ in (1). Inspired by [18], we need to find the transformation to convert system (1) into the target system that is a disturbance free system, and at the same, both the controller and the output keep invariant. For this purpose, we suppose that the auxiliary trajectory satisfies the following system:

$$
\begin{aligned}
\phi_{t}(x, t) & =\phi_{x x}(x, t), \quad x \in(0,1), t>0, \\
\phi_{x}(0, t) & =Q v(t), \quad t \geq 0, \\
\phi(0, t) & =P_{1} v(t), \quad t \geq 0,
\end{aligned}
$$

where $P_{1}$ is an $n$-dimensional row vector such that $\phi(1, t)=0$. Inspired by [18] and [19, Chapter 12], again, we try to find a special solution of system (5) in the following form:

$$
\phi(x, t)=\sum_{n=0}^{\infty} \alpha_{n}(t) \frac{x^{n}}{n !}, \quad x \in[0,1], t \geq 0 .
$$

Inserting (6) into system (5), we have

$$
\begin{aligned}
& \dot{\alpha}_{n}(t)=\alpha_{n+2}(t), \quad n=0,1,2 \cdots \\
& \alpha_{0}(t)=P_{1} v(t), \\
& \alpha_{1}(t)=Q v(t),
\end{aligned}
$$

which leads to

$$
\begin{gathered}
\phi(x, t)=\sum_{n=0}^{\infty} P_{1} v^{n}(t) \frac{x^{2 n}}{(2 n) !}+\sum_{n=0}^{\infty} Q v^{n}(t) \frac{x^{2 n+1}}{(2 n+) !} \\
=P_{1}\left(\sum_{n=0}^{\infty} \frac{G^{n} x^{2 n}}{(2 n) !}\right) v(t)+Q\left(\sum_{n=0}^{\infty} \frac{G^{n} x^{2 n+1}}{(2 n+1) !}\right) v(t) \\
=\left[P_{1} \cosh \left(x G^{1 / 2}\right)+Q G^{-1 / 2} \sinh \left(x G^{1 / 2}\right)\right] v(t) .
\end{gathered}
$$

Since $\phi(1, t)=0$, we get

$$
\begin{aligned}
\phi(1, t) & =\left[P_{1} \cosh \left(G^{1 / 2}\right)+Q G^{-1 / 2} \sinh \left(G^{1 / 2}\right)\right] v(t) \\
& =0
\end{aligned}
$$

or equivalently

$$
P_{1}=-Q G^{-1 / 2} \tanh \left(G^{1 / 2}\right) .
$$


We suppose

$$
\begin{aligned}
& \psi_{t t}(x, t)=\psi_{x x}(x, t), \quad x \in(0,1), t>0, \\
& \psi_{x}(1, t)=c_{0} \psi_{t}(1, t), \quad t \geq 0, \\
& \psi(1, t)=P_{2} v(t), \quad t \geq 0,
\end{aligned}
$$

where $P_{2}$ is an $n$-dimensional row vector such that $\psi_{x}(0, t)=$ $c_{1} \phi(0, t)-c_{2} \psi(0, t)$. We try to find a special solution of system (11) in the following form:

$$
\psi(x, t)=\sum_{n=0}^{\infty} \beta_{n}(t) \frac{(x-1)^{n}}{n !}, \quad x \in[0,1], t \geq 0 .
$$

Inserting (11) into system (12), we have

$$
\begin{aligned}
& \ddot{\beta}_{n}(t)=\beta_{n+2}(t), \quad n=0,1,2 \ldots \\
& \beta_{0}(t)=P_{2} v(t), \\
& \beta_{1}(t)=c_{0} P_{2} G v(t),
\end{aligned}
$$

which leads to

$$
\begin{aligned}
\psi(x, t)= & P_{2} \cosh ((x-1) G) v(t) \\
& +c_{0} P_{2} \sinh ((x-1) G) v(t) .
\end{aligned}
$$

Since $\psi_{x}(0, t)=c_{1} \phi(0, t)-c_{2} \psi(0, t)$, we get

$$
\begin{gathered}
\psi_{x}(0, t)=P_{2} \sinh (-G) G v(t)+c_{0} P_{2} \cosh (-G) G v(t) \\
=c_{1} P_{1} v(t) \\
\quad-c_{2}\left(P_{2} \cosh (-G) v(t)+c_{0} P_{2} \sinh (-G) v(t)\right),
\end{gathered}
$$

or equivalently

$$
P_{2}=c_{1} P_{1} \cdot H^{-1}
$$

where

$$
\begin{aligned}
H= & \sinh (-G) G+c_{0} \cosh (-G) G+c_{2} \cosh (-G) \\
& +c_{2} c_{0} \sinh (-G) .
\end{aligned}
$$

If we let

$$
\begin{aligned}
& \rho(x, t)=u(x, t)-\psi(x, t), \quad x \in[0,1], t \geq 0, \\
& \varphi(x, t)=z(x, t)-\phi(x, t), \quad x \in[0,1], t \geq 0,
\end{aligned}
$$

then, by (1), (5), and (11)

$$
\begin{aligned}
\rho_{t t}(x, t) & =\rho_{x x}(x, t), \quad x \in(0,1), \quad t>0, \\
\rho_{x}(1, t) & =c_{0} \rho_{t}(1, t), \quad t \geq 0, \\
\rho_{x}(0, t) & =c_{1} \varphi(0, t)-c_{2} \rho(0, t), \quad t \geq 0, \\
\varphi_{t}(x, t) & =\varphi_{x x}(x, t), \quad x \in(0,1), \quad t>0, \\
\varphi_{x}(0, t) & =0, \quad t \geq 0, \\
\varphi(1, t) & =U(t), \quad t \geq 0, \\
\dot{v}(t) & =G v(t), \quad t \geq 0,
\end{aligned}
$$

and

$$
\begin{aligned}
Y_{e}(t) & =Y_{r e f}(t)-\psi(1, t)-\rho(1, t) \\
& =\left(F-P_{2}\right) v(t)-\rho(1, t) .
\end{aligned}
$$

Comparing system (1) with system (19), one can find that the disturbance $Q v(t)$ in system (1) has been canceled in system (19). The next objective is to stabilize the regulation error $Y_{e}$. To this end, we will apply the trajectory planning again to bring the external signal $\left(F-P_{2}\right) v(t)$ into the control channel such that the regulation error $Y_{e}$ translates into a state of the target system. Naturally, we only need to stabilize the target system to achieve our goal.

We suppose that the trajectory satisfies the following system:

$$
\begin{aligned}
& \eta_{t t}(x, t)=\eta_{x x}(x, t), \quad x \in(0,1), \quad t>0, \\
& \eta_{x}(1, t)=-c_{0} \eta_{t}(1, t), \quad t \geq 0, \\
& \eta_{x}(0, t)=c_{1} \mu(0, t)-c_{2} \eta(0, t), \quad t \geq 0, \\
& \mu_{t}(x, t)=\mu_{x x}(x, t), \quad x \in(0,1), t>0, \\
& \mu_{x}(0, t)=0, \quad t \geq 0, \\
& \mu(1, t)=P_{3} v(t), \quad t \geq 0,
\end{aligned}
$$

where $P_{3}$ is an $n$-dimensional row vector that will be determined later.

Suppose that the " $\rho$-part" of (21) satisfies

$$
\begin{gathered}
\eta_{t t}(x, t)=\eta_{x x}(x, t), \quad x \in(0,1), t>0, \\
\eta_{x}(1, t)=-c_{0} \eta_{t}(1, t), \quad t \geq 0, \\
\eta(1, t)=\left(F-P_{2}\right) v(t), \quad t \geq 0 .
\end{gathered}
$$

Taking (5), (6), (7)n and (8) into account, system (22) admits a special solution

$$
\begin{aligned}
\eta(x, t)= & \left(F-P_{2}\right) \cosh ((x-1) G) v(t) \\
& +c_{0}\left(F-P_{2}\right) \sinh ((x-1) G) v(t) .
\end{aligned}
$$

It implies that

$$
\begin{aligned}
\eta_{x}(0, t)= & \left(F-P_{2}\right) \sinh (-G) G v(t) \\
& +c_{0}\left(F-P_{2}\right) \cosh (-G) G v(t), \\
\eta_{x}(0, t)= & c_{1} \mu(0, t)-c_{2} \eta(0, t) .
\end{aligned}
$$

From (24), we have

$$
\mu(0, t)=\frac{1}{c_{1}}\left(F-P_{2}\right) \cdot H \cdot v(t),
$$

where $H$ is defined by (17)

Suppose that the " $\mu$-part" of (21) satisfies

$$
\begin{aligned}
& \mu_{t}(x, t)=\mu_{x x}(x, t), \quad x \in(0,1), t>0, \\
& \mu_{x}(0, t)=0, \quad t \geq 0, \\
& \mu(0, t)=\frac{1}{c_{1}}\left(F-P_{2}\right) \cdot H \cdot v(t), \quad t \geq 0 .
\end{aligned}
$$


We will find a special solution for " $\mu$-part" of (26) that takes the form

$$
\mu(x, t)=\sum_{n=0}^{\infty} \xi_{n}(t) \frac{x^{n}}{n !}, \quad x \in[0,1], t \geq 0 .
$$

Hence,

$$
\mu(x, t)=\frac{1}{c_{1}}\left(F-P_{2}\right) \cdot H \cdot \cosh \left(x G^{1 / 2}\right) v(t) .
$$

More specifically,

$$
\mu(1, t)=P_{3} v(t)=\frac{1}{c_{1}}\left(F-P_{2}\right) \cdot H \cdot \cosh \left(G^{1 / 2}\right) v(t) .
$$

Or equivalently

$$
P_{3}=\frac{1}{c_{1}}\left(F-P_{2}\right) \cdot H \cdot \cosh \left(G^{1 / 2}\right) .
$$

If we let

$$
\begin{aligned}
& \varepsilon(x, t)=\eta(x, t)-\rho(x, t), \\
& \gamma(x, t)=\mu(x, t)-\varphi(x, t),
\end{aligned}
$$

then, by (19) and (21),

$$
\begin{aligned}
& \varepsilon_{t t}(x, t)=\varepsilon_{x x}(x, t), \quad x \in(0,1), t>0, \\
& \varepsilon_{x}(1, t)=-c_{0} \varepsilon_{t}(1, t), \quad t \geq 0, \\
& \varepsilon_{x}(0, t)=c_{1} \gamma(0, t)+c_{2} \varepsilon(0, t), \quad t \geq 0, \\
& \gamma_{t}(x, t)=\gamma_{x x}(x, t), \quad x \in(0,1), t>0, \\
& \gamma_{x}(0, t)=0, \quad t \geq 0, \\
& \gamma(1, t)=P_{3} v(t)-U(t), \quad t \geq 0,
\end{aligned}
$$

and

$$
\begin{aligned}
Y_{e}(t) & =\left(F-P_{2}\right) v(t)-\rho(1, t)=\eta(1, t)-\varphi(1, t) \\
& =\varepsilon(1, t) .
\end{aligned}
$$

\section{Controller Design}

Equation (33) implies that we only need to stabilize system (32) to achieve output regulation (1) without input delay. In this way, the controller with $\tau=0$ can be designed easily

$$
U(t)=P_{3} v(t)=\frac{1}{c_{1}}\left(F-P_{2}\right) \cdot H \cdot \cosh \left(G^{1 / 2}\right) v(t),
$$

under which, we get the closed-loop system of (34)

$$
\begin{aligned}
\dot{v}(t) & =G v(t), \quad t \geq 0, \\
u_{t t}(x, t) & =u_{x x}(x, t), \quad x \in(0,1), \quad t>0, \\
u_{x}(1, t) & =-c_{0} u_{t}(1, t), \quad t \geq 0, \\
u_{x}(0, t) & =c_{1} z(0, t)+c_{2} u(0, t), \quad t \geq 0,
\end{aligned}
$$

$$
\begin{aligned}
& z_{t}(x, t)=z_{x x}(x, t), \quad x \in(0,1), t>0, \\
& z_{x}(0, t)=d(t), \quad t \geq 0, \\
& z(1, t)=\frac{1}{c_{1}}\left(F-P_{2}\right) \cdot H \cdot \cosh \left(G^{1 / 2}\right) v(t), \quad t \geq 0, \\
& u(x, 0)=u_{0}(x), \quad x \in[0,1], \\
& u_{\mathrm{t}}(x, 0)=u_{1}(x), \quad x \in[0,1], \\
& z(x, 0)=z_{0}(x), \quad x \in[0,1] .
\end{aligned}
$$

Theorem 1. For any initial state $(v(0), u(\cdot, 0), z(\cdot, 0)) \in \mathscr{H}$, system (35) has unique solution $(v(t), u(\cdot, t), z(\cdot, t)) \in C([0, \infty)$; $\mathscr{H})$ such that, for any $t_{0}>0$,

$$
\left|Y_{e}(t)\right| \leq L_{1} e^{-\omega_{1} t}, \quad \forall t \geq t_{0},
$$

where $L_{1}$ and $\omega_{1}$ are two positive constants. Moreover, the state of the closed-loop system (35) is uniformly bounded

$$
\sup _{t \in[0, \infty]}\|v(t), u(\cdot, t), z(\cdot, t)\|_{\mathscr{H}}<+\infty .
$$

Proof. We first consider the following transformed system:

$$
\begin{aligned}
& \varepsilon_{t t}(x, t)=\varepsilon_{x x}(x, t), \quad x \in(0,1), t>0, \\
& \varepsilon_{x}(1, t)=-c_{0} \varepsilon_{t}(1, t), \quad t \geq 0, \\
& \varepsilon_{x}(0, t)=c_{1} \gamma(0, t)+c_{2} \varepsilon(0, t), \quad t \geq 0, \\
& \gamma_{t}(x, t)=\gamma_{x x}(x, t), \quad x \in(0,1), t>0, \\
& \gamma_{x}(0, t)=0, \quad t \geq 0, \\
& \gamma(1, t)=0, \quad t \geq 0,
\end{aligned}
$$

with the initial state

$$
\begin{array}{ll}
\varepsilon(x, 0)=\psi(x, 0)+\eta(x, 0)-u(x, 0), & x \in[0,1], \\
\gamma(x, 0)=\mu(x, 0)+\phi(x, 0)-z(x, 0), & x \in[0,1],
\end{array}
$$

where $\psi, \eta, \mu$, and $\phi$ are defined by (14), (23), (28), and (8), respectively. As system (38) is a cascade of the heat equation and the wave equation and the " $\gamma$-subsystem" of (38) is independent of the " $\varepsilon$-subsystem", it is well known that there exists a unique solution $(\varepsilon(\cdot, t), \gamma(\cdot, t)) \in C\left([0, \infty) ; H^{1}(0,1) \times\right.$ $\left.L^{2}(0,1)\right)$ to system $(38)$.

Consider the Lyapunov function

$$
\begin{aligned}
V= & \frac{1}{2}\left(\int_{0}^{1} \varepsilon_{x}^{2} d x+\int_{0}^{1} \varepsilon_{t}^{2} d x+c_{2} \varepsilon^{2}(0)\right) \\
& +\delta \int_{0}^{1}(1+x) \varepsilon_{x}(x) \varepsilon_{t}(x) d x+\frac{1}{2} \int_{0}^{1} \gamma^{2}(x) d x .
\end{aligned}
$$

Using the Cauchy-Schwarz and Yong's inequalities, there exist $m_{1}, m_{2}$ such that

$$
m_{1} \Pi \leq V \leq m_{2} \Pi \text {, }
$$


where

$$
\Pi=\int_{0}^{1} \varepsilon_{x}^{2} d x+\int_{0}^{1} \varepsilon_{t}^{2} d x+\varepsilon^{2}(0)+\int_{0}^{1} \gamma^{2}(x) d x .
$$

Therefore $V$ is positive definite.

The derivative of $V$ along the solution of system (38) is

$$
\begin{aligned}
& \frac{d V}{d t}=\int_{0}^{1} \varepsilon_{x} \varepsilon_{t x} d x+\int_{0}^{1} \varepsilon_{t} \varepsilon_{t t} d x+c_{2} \varepsilon(0) \varepsilon_{t}(0) \\
& +\delta \int_{0}^{1}(1+x)\left(\varepsilon_{x t} \varepsilon_{t}+\varepsilon_{x} \varepsilon_{t t}\right) d x+\int_{0}^{1} \gamma \gamma_{t} d x \\
& =\int_{0}^{1} \varepsilon_{x} \varepsilon_{t x} d x+\int_{0}^{1} \varepsilon_{t} \varepsilon_{x x} d x \\
& +\left(\varepsilon_{x}(0, t)-c_{1} \gamma(0, t)\right) \varepsilon_{t}(0) \\
& +\delta \int_{0}^{1}(1+x)\left(\varepsilon_{x t} \varepsilon_{t}+\varepsilon_{x} \varepsilon_{x x}\right) d x+\int_{0}^{1} \gamma \gamma_{x x} d x \\
& =\int_{0}^{1} \varepsilon_{x} \varepsilon_{t x} d x-\int_{0}^{1} \varepsilon_{x t} \varepsilon_{x} d x+\varepsilon_{x}(1) \varepsilon_{t}(1) \\
& -c_{1} \gamma(0, t) \varepsilon_{t}(0) \\
& +\delta \int_{0}^{1}(1+x)\left(\varepsilon_{x t} \varepsilon_{t}+\varepsilon_{x} \varepsilon_{x x}\right) d x-\int_{0}^{1} \gamma_{x}^{2} d x \\
& \leq-c_{0} \varepsilon_{t}^{2}(1)-c_{1} \gamma(0, t) \varepsilon_{t}(0) \\
& +\left.\frac{\delta}{2}\left[(1+x)\left(\varepsilon_{t}^{2}+\varepsilon_{x}^{2}\right)\right]\right|_{0} ^{1}-\frac{\delta}{2} \int_{0}^{1}\left(\varepsilon_{t}^{2}+\varepsilon_{x}^{2}\right) d x \\
& -\int_{0}^{1} \gamma_{x}^{2} d x \\
& \leq-\left(c_{0}-\delta-\delta c_{0}^{2}\right) \varepsilon_{t}^{2}(1)-\left(\frac{\delta}{2}-\frac{c_{1}}{2}\right) \varepsilon_{t}^{2}(0) \\
& -\frac{\delta}{2} \int_{0}^{1}\left(\varepsilon_{t}^{2}+\varepsilon_{x}^{2}\right) d x \\
& -\left(1-\frac{c_{1}}{2}-\delta c_{1} c_{2}\right) \int_{0}^{1} \gamma^{2} d x \\
& -\left(\delta c_{2}^{2}-2 \delta c_{1} c_{2}\right) \varepsilon^{2}(0)
\end{aligned}
$$

which is negative definite for

$$
\begin{aligned}
0 & <c_{1}<\frac{1}{2}, \\
2 c_{1} & <c_{2}<1, \\
c_{1} & <\delta<\frac{c_{0}}{1+c_{0}^{2}} .
\end{aligned}
$$

It follows from (40) and (41) that

$$
\Pi(t) \leq M e^{-t / M} \Pi(0)
$$

for some possibly large $M$, which proves the exponential stability of the " $\varepsilon-\gamma$ " system.
On the other hand, since the " $v$-subsystem" of (35) is independent of the other subsystems, it admits a unique solution $v(t) \in C\left([0, \infty) ; \mathbb{C}^{n}\right)$.

We define

$$
\begin{aligned}
& u(x, t)=\psi(x, t)+\eta(x, t)-\varepsilon(x, t), \\
& x \in[0,1], t \geq 0, \\
& z(x, t)=\mu(x, t)+\phi(x, t)-\gamma(x, t),
\end{aligned}
$$

$$
x \in[0,1], t \geq 0,
$$

where $\psi, \eta, \mu$, and $\phi$ are defined by (14), (23), (28), and (8), respectively. Now, it is easy to verify that such a defined $(v(t), u(\cdot, t), z(\cdot, t)) \in C([0, \infty) ; \mathscr{H})$ is a solution of system (35). Moreover, the uniformly bounded (37) holds due to (45), (46), and the fact that $G$ is dissipative.

The proof is complete.

\section{Observer Design}

In this section, one will design the observer for $v(t)$. According to the ideal of [18], the state observer can be designed as follows:

$$
\begin{aligned}
& \dot{\hat{v}}(t)=G \widehat{v}(t)+c_{3}\left(F-P_{2}\right)^{*} \\
& \quad \cdot\left[-\dot{y}_{e}(t)-\widehat{\rho}_{t}(1, t)+\left(F-P_{2}\right) G \widehat{v}(t)\right], \quad t \geq 0, \\
& \widehat{\rho}_{t t}(x, t)=\widehat{\rho}_{x x}(x, t), \quad x \in(0,1), \quad t>0, \\
& \widehat{\rho}_{x}(1, t)=-c_{0} \widehat{\rho}_{t}(1, t)-c_{3} y_{e}(t)-c_{3} \widehat{\rho}(1, t) \\
& \quad+c_{3}\left(F-P_{2}\right) \widehat{v}(t), \quad t \geq 0, \\
& \widehat{\rho}_{x}(0, t)=c_{1} \widehat{\varphi}(0, t)+c_{2} \widehat{\rho}(0, t), \quad t \geq 0, \\
& \widehat{\varphi}_{t}(x, t)=\widehat{\varphi}_{x x}(x, t), \quad x \in(0,1), t>0, \\
& \widehat{\varphi}_{x}(0, t)=0, \quad t \geq 0, \\
& \widehat{\varphi}(1, t)=U(t), \quad t \geq 0,
\end{aligned}
$$

where $0<c_{3}<1$ is the tuning parameter and $K=F-P_{1}$ is the conjugate transpose of $K^{*}$.

Let

$$
\begin{aligned}
& \widetilde{v}(x, t)=v(x, t)-\widehat{v}(x, t), \\
& \widetilde{\rho}(x, t)=\rho(x, t)-\widehat{\rho}(x, t), \\
& \widetilde{\varphi}(x, t)=\varphi(x, t)-\widehat{\varphi}(x, t) .
\end{aligned}
$$

We have the error system as follows:

$$
\dot{\tilde{v}}(t)=G \widetilde{v}(t)-c_{3} K^{*} \widetilde{\rho}_{t}(1, t)+c_{3} K^{*} K G \widetilde{v}(t),
$$

$$
t \geq 0
$$

$\tilde{\rho}_{t t}(x, t)=\tilde{\rho}_{x x}(x, t), \quad x \in(0,1), t>0$, 


$$
\begin{aligned}
& \tilde{\rho}_{x}(1, t)=-c_{0} \tilde{\rho}_{t}(1, t)-c_{3} \widetilde{\rho}(1, t)-c_{3} K \widetilde{v}(t), \quad t \geq 0, \\
& \tilde{\rho}_{x}(0, t)=c_{1} \widetilde{\varphi}(0, t)+c_{2} \tilde{\rho}(0, t), \quad t \geq 0, \\
& \tilde{\varphi}_{t}(x, t)=\widetilde{\varphi}_{x x}, \quad x \in(0,1), t>0 \\
& \widetilde{\varphi}_{x}(0, t)=0, \quad t \geq 0 \\
& \widetilde{\varphi}(1, t)=0, \quad t \geq 0
\end{aligned}
$$

where $c_{3}$ is the tuning parameter and $K=F-P_{2}$ is the conjugate transpose of $K^{*}$.

Moreover, we have the following theorem.

Theorem 2. Suppose that $G+c_{3} K^{*} K G$ is Hurwitz. Then solution of system (49) is asymptotically stable.

Proof. Since $G+c_{3} K^{*} K$ is Hurwitz, there exists a positive constant $a_{1}$ such that

$$
\left(G+c_{3} K^{*} K\right)|\widetilde{v}|^{2} \leq-a_{1}\|\widetilde{v}\|_{\mathbb{C}^{n}}^{2}
$$

Consider the following Lyapunov function.

$$
\begin{aligned}
V_{1}= & \frac{1}{2}|\widetilde{v}|^{2} \\
& +\frac{1}{2}\left(\int_{0}^{1} \widetilde{\rho}_{x}^{2} d x+\int_{0}^{1} \widetilde{\rho}_{t}^{2} d x+c_{2} \widetilde{\rho}^{2}(0)+c_{3} \widetilde{\rho}^{2}(1)\right) \\
& +\frac{1}{2} \int_{0}^{1} \widetilde{\varphi}^{2}(x) d x
\end{aligned}
$$

The derivative of $V_{1}$ along the solution of system (49) is

$$
\begin{aligned}
& \frac{d V_{1}}{d t}=\tilde{v} \dot{\bar{v}}+\int_{0}^{1} \tilde{\rho}_{x} \tilde{\rho}_{t x} d x+\int_{0}^{1} \tilde{\rho}_{t} \tilde{\rho}_{t t} d x+c_{2} \tilde{\rho}(0) \tilde{\rho}_{t}(0) \\
& +\int_{0}^{1} \widetilde{\varphi} \widetilde{\varphi}_{t} d x+c_{3} \widetilde{\rho}(1) \widetilde{\rho}_{t}(1) \\
& =\left(G+c_{3} K^{*} K\right)|\widetilde{v}|^{2}(t)+c_{3} K^{*} \tilde{\rho}_{t}(1, t) \widetilde{v}(t) \\
& +\int_{0}^{1} \widetilde{\rho}_{x} \widetilde{\rho}_{t x} d x+\int_{0}^{1} \widetilde{\rho}_{t} \widetilde{\rho}_{x x} d x+c_{2} \widetilde{\rho}(0) \widetilde{\rho}_{t}(0) \\
& +\int_{0}^{1} \tilde{\varphi} \widetilde{\varphi}_{t} d x+c_{3} \tilde{\rho}(1) \tilde{\rho}_{t}(1) \\
& =\left(G+c_{3} K^{*} K\right)|\widetilde{v}|^{2}(t) \\
& +c_{3} K^{*} \tilde{\rho}_{t}(1, t) v(t)+\int_{0}^{1} \tilde{\rho}_{x} \tilde{\rho}_{t x} d x+\left.\widetilde{\rho}_{t} \tilde{\rho}_{x}\right|_{0} ^{1} \\
& -\int_{0}^{1} \widetilde{\rho}_{x t} \widetilde{\rho}_{x} d x+\left(\widetilde{\rho}_{x}(0)-c_{1} \widetilde{\varphi}(0)\right) \tilde{\rho}_{t}(0) \\
& +\int_{0}^{1} \tilde{\varphi} \widetilde{\varphi}_{x x} d x+c_{3} \tilde{\rho}(1) \tilde{\rho}_{t}(1)
\end{aligned}
$$

$$
\begin{aligned}
\leq & \left(G+c_{3} K^{*} K\right)|\widetilde{v}|^{2}(t)+c_{3} K^{*} \tilde{\rho}_{t}(1) \widetilde{v}(t) \\
& -c_{0}\left|\widetilde{\rho}_{t}(1)\right|^{2}-c_{3} \widetilde{\rho}(1) \tilde{\rho}_{t}(1)-c_{3} K \widetilde{v}(t) \tilde{\rho}_{t}(1) \\
& -c_{1} \widetilde{\varphi}(0) \widetilde{\rho}_{t}(0)-\int_{0}^{1} \widetilde{\varphi}_{x}^{2} d x+c_{3} \widetilde{\rho}(1) \tilde{\rho}_{t}(1) \\
\leq & -a_{1}\|v\|_{\mathbb{C}^{n}}^{2}-c_{0}\left|\widetilde{\rho}_{t}(1)\right|^{2} \\
& +\frac{c_{1}}{2}\left(|\widetilde{\varphi}(0)|^{2}+\left|\widetilde{\rho}_{t}(0)\right|^{2}\right)-\int_{0}^{1} \widetilde{\varphi}_{x}^{2} d x \\
\leq & -a_{1}\|v\|_{\mathbb{C}^{n}}^{2}-\left(c_{0}-\frac{c_{1}}{2}\right)\left|\widetilde{\rho}_{t}(1)\right|^{2} \\
& -\left(1-\frac{c_{1}}{2}\right) \int_{0}^{1} \widetilde{\varphi}_{x}^{2} d x
\end{aligned}
$$

which is negative definite for

$$
\begin{aligned}
& c_{1}<2, \\
& c_{0}>\frac{c_{1}}{2} .
\end{aligned}
$$

This completes the proof of the theorem.

Theorem 3. Suppose that $G+c_{3} K^{*} K$ is Hurwitz. Then, for any initial state $(v(0), \rho(\cdot, 0), \varphi(\cdot, 0), \widehat{v}(0), \widehat{\varphi}(\cdot, 0), \widehat{z}(\cdot, 0)) \in \mathscr{H}^{2}$ and $U \in H_{l o c}^{1}(0, \infty)$, system (14)-(47) admits a unique solution

$$
\begin{aligned}
& (v(t), \rho(\cdot, t), \varphi(\cdot, t), \widehat{v}(t), \widehat{\rho}(\cdot, t), \widehat{\varphi}(\cdot, t)) \\
& \quad \in C\left([0, \infty) ; \mathscr{H}^{2}\right)
\end{aligned}
$$

such that

$$
\begin{aligned}
& \|(v(t)-\widehat{v}(t), \rho(\cdot, t)-\hat{\rho}(\cdot, t), \varphi(\cdot, t)-\widehat{\varphi}(\cdot, t))\|_{\mathscr{H}} \\
& \quad \longrightarrow 0 \text { as } t \longrightarrow \infty .
\end{aligned}
$$

Proof. It is well known that, for any $(v(0), \rho(\cdot, 0), \varphi(\cdot, 0)) \in \mathscr{H}$ and $U \in H_{l o c}^{1}(0, \infty)$, system (19) admits a unique solution

$$
(v(t), \rho(\cdot, t), \varphi(\cdot, t)) \in C([0, \infty) ; \mathscr{H}) .
$$

From Theorem 2, we know that system (49) admits a unique solution $(\widetilde{v}(t), \widetilde{\rho}(\cdot, t), \widetilde{\varphi}(\cdot, t)) \in C([0, \infty) ; \mathscr{H})$ with initial state $(\widetilde{v}(0), \widetilde{\rho}(\cdot, 0), \widetilde{\varphi}(\cdot, 0))=(v(0)-\widehat{v}(0), \rho(\cdot, 0)-\widehat{\rho}(\cdot, 0), \varphi(\cdot, 0)-$ $\widehat{\varphi}(\cdot, 0)$, ) such that

$$
\|(\widetilde{v}(t), \widetilde{\varphi}(\cdot, t), \widetilde{z}(\cdot, t))\|_{\mathscr{H}} \longrightarrow 0 \quad \text { as } t \longrightarrow \infty .
$$

Then, we define

$$
\begin{aligned}
\widehat{v}(t) & =v(t)-\widetilde{v}(t), \quad x \in[0,1], \\
\widehat{\rho}(x, t) & =\rho(x, t)-\tilde{\rho}(x, t), \quad x \in[0,1], \\
\widehat{\varphi}(x, t) & =\varphi(x, t)-\widetilde{\varphi}(x, t), \quad x \in[0,1],
\end{aligned}
$$

Hence, $(v(t), \rho(\cdot, t), \varphi(\cdot, t), \widehat{v}(t), \widehat{\rho}(\cdot, t), \widehat{\varphi}(\cdot, t)) \in C\left([0, \infty) ; \mathscr{H}^{2}\right)$ is a solution of system (19)-(47). Moreover, by (57) and (58), we can see (55) holds. The proof is complete. 


\section{The Uniform Boundedness of the Loop System}

Replacing $v$ with $\widehat{v}$, one will obtain the following closedsystem of (1):

$$
\begin{aligned}
& \dot{v}(t)=G v(t), \quad t \geq 0, \\
& u_{t t}(x, t)=u_{x x}(x, t), \quad x \in(0,1), t>0, \\
& u_{x}(0, t)=c_{1} z(0, t)+c_{2} u(0, t), \quad t \geq 0, \\
& u_{x}(1, t)=-c_{0} u_{t}(1, t), \quad t \geq 0, \\
& z_{t}(x, t)=z_{x x}(x, t), \quad x \in(0,1), t>0, \\
& z_{x}(0, t)=d(t), \quad t \geq 0, \\
& z(1, t)=U(t), \quad t \geq 0, \\
& \dot{\hat{v}}(t)=G \widehat{v}(t)+c_{3}\left(F-P_{2}\right)^{*} \\
& \cdot\left[-\dot{y}_{e}(t)-\hat{\rho}_{t}(1, t)+\left(F-P_{2}\right) G \widehat{v}(t)\right], \quad t \geq 0, \\
& \hat{\rho}_{t t}(x, t)=\hat{\rho}_{x x}(x, t), \quad x \in(0,1), t>0, \\
& \hat{\rho}_{x}(1, t)=-c_{0} \hat{\rho}_{t}(1, t)-c_{3} y_{e}(t)-c_{3} \hat{\rho}(1, t) \\
& +c_{3}\left(F-P_{2}\right) \hat{v}(t), \quad t \geq 0, \\
& \widehat{\rho}_{x}(0, t)=c_{1} \widehat{\varphi}(0, t)+c_{2} \widehat{\rho}(0, t), \quad t \geq 0, \\
& \widehat{\varphi}_{t}(x, t)=\widehat{\varphi}_{x x}(x, t), \quad x \in(0,1), t>0, \\
& \widehat{\varphi}_{x}(0, t)=0, \quad t \geq 0, \\
& \widehat{\varphi}(1, t)=U(t), \quad t \geq 0 .
\end{aligned}
$$

Moreover, one has the following theorem.

Theorem 4. Suppose that $G+c_{3} K^{*} K$ is Hurwitz. Then, for any initial state $(v(0), u(\cdot, 0), z(\cdot, 0), \widehat{v}(0), \widehat{\rho}(\cdot, 0), \widehat{\varphi}(\cdot, 0)) \in \mathscr{H}^{2}$, the closed-loop (59) admits a unique solution

$$
\begin{aligned}
& (v(t), u(\cdot, t), z(\cdot, t), \widehat{v}(t), \widehat{\rho}(\cdot, t), \widehat{\varphi}(\cdot, t)) \\
& \quad \in C\left([0, \infty) ; \mathscr{H}^{2}\right)
\end{aligned}
$$

such that

$$
\|d(t)-Q \widehat{v}(t)\|_{C^{n}}+\left|Y_{e}(t)\right| \longrightarrow 0 \text { as } t \longrightarrow \infty .
$$

If we assume further that $G$ is dissipative, then the state of system is uniformly bounded

$$
\begin{aligned}
& \sup _{t \in[0, \infty)}\|(v(t), u(\cdot, t), z(\cdot, t), \widehat{v}(t), \widehat{\rho}(\cdot, t), \widehat{\varphi}(\cdot, t))\|_{\mathscr{H}^{2}} \\
& \quad<\infty
\end{aligned}
$$

Proof. According to Theorems 1 and 3, system (59) admits a unique solution

$$
\begin{aligned}
& (v(t), u(\cdot, t), z(\cdot, t), \widehat{v}(t), \widehat{\varphi}(\cdot, t), \widehat{z}(\cdot, t)) \\
& \quad \in C\left([0, \infty) ; \mathscr{H}^{2}\right)
\end{aligned}
$$

with initial state $(v(0), u(\cdot, 0), z(\cdot, 0), \widehat{v}(0), \widehat{\varphi}(\cdot, 0), \widehat{z}(\cdot, 0)) \quad \epsilon$ $\mathscr{H}^{2}$.

Next, We define

$$
\begin{aligned}
u(x, t) & =\psi(x, t)+\eta(x, t)-\varepsilon(x, t), \quad x \in[0,1], \\
z(x, t) & =\mu(x, t)+\phi(x, t)-\gamma(x, t), \quad x \in[0,1], \\
\widehat{\varphi}(x, t) & =\varphi(x, t)-\widetilde{\varphi}(x, t), \quad x \in[0,1], \\
\widehat{\rho}(x, t) & =\rho(x, t)-\tilde{\rho}(x, t), \quad x \in[0,1], \\
\widehat{v}(t) & =v(t)-\widetilde{v}(t), \quad x \in[0,1],
\end{aligned}
$$

and, by Theorems 1 and 2, it is easy to check

$$
\begin{aligned}
& \sup _{t \in[0, \infty)}\|(v(t), u(\cdot, t), z(\cdot, t), \widehat{v}(t), \widehat{\rho}(\cdot, t), \widehat{\varphi}(\cdot, t))\|_{\mathscr{H}^{2}} \\
& \quad<\infty
\end{aligned}
$$

By (36) and (56), we can see (61) holds. The proof is complete.

\section{Numerical Simulation}

In this section, one presents some numerical simulations to validate our theory results. We give the numerical simulation results for system $(v, u, \widehat{v}, \widehat{\varphi})$ which is governed by (59). The corresponding parameters are chosen as

$$
G=\left(\begin{array}{cc}
0 & 1 \\
-4 & 0
\end{array}\right)
$$

and

$$
\begin{aligned}
& F=(1,0), \\
& Q=(0,1), \\
& c_{0}=1.3 .
\end{aligned}
$$

The initial states are selected as

$$
\begin{aligned}
u(x, 0) & =0.01 \cos (2 \pi x), \\
\widehat{\varphi}(x, 0) & =\sin (2 \pi x), \\
v & =(0,2) \\
\widehat{v} & =(0,-3) .
\end{aligned}
$$

The time step and space step are taken as $0.001 \mathrm{~s}$ and $0.05 \mathrm{~s}$.

The solution of the closed-loop system (59) is plotted in Figure 1. The output tracking and the disturbance estimation are plotted in Figure 2. In Figures 1 and 2, we choose $c_{1}=0.1$, $c_{2}=0.7, c_{3}=0.8$.

Figure 1 displays the numerical results for closed-loop system in the noncollocated error feedback output regulation case. Figures 1(a) and 1(b) display the states of the controlled system $u(x, t), z(x, t)$. The states of the observer $\hat{\rho}(x, t), \widehat{\varphi}(x, t)$ are plotted in Figures 1(c) and 1(d). It is obvious that all states are bounded. 


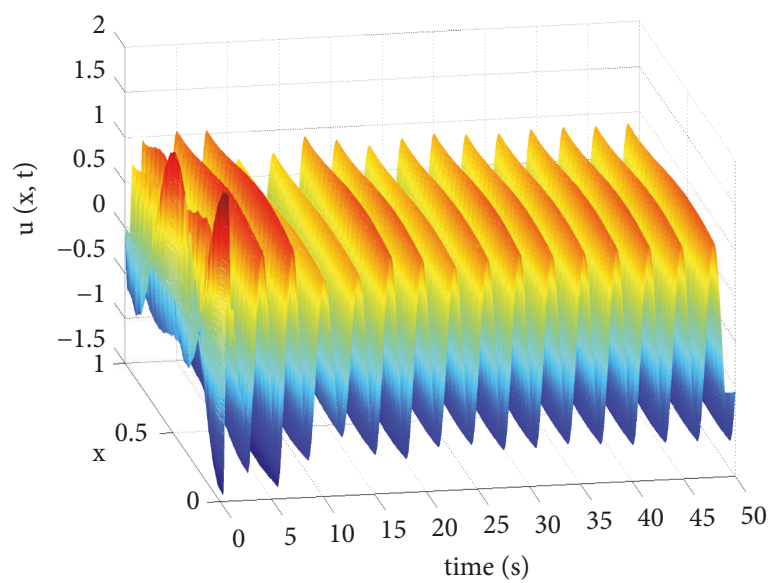

(a) $u(x, t)$

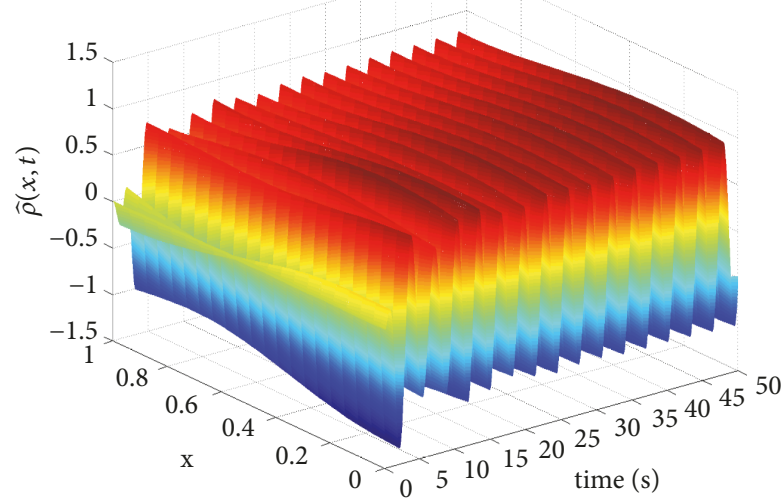

(c) $\hat{\rho}(x, t)$

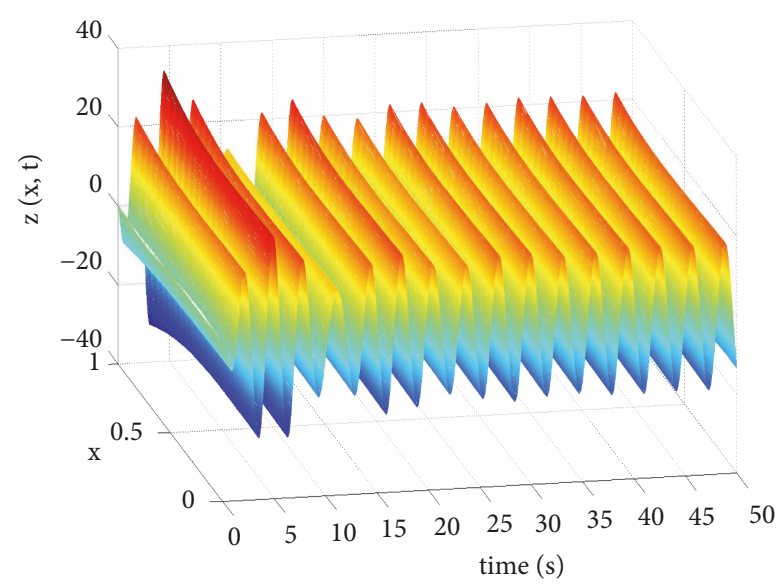

(b) $z(x, t)$

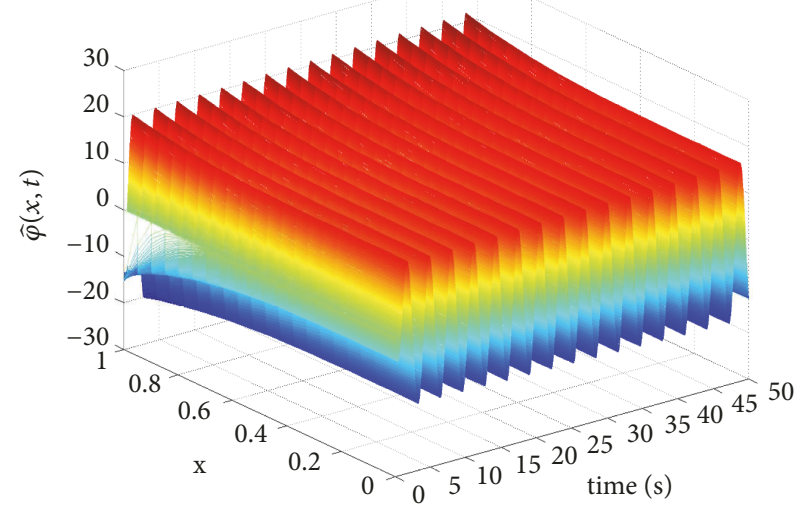

(d) $\hat{\varphi}(x, t)$

FIGURE 1: The solution of closed-loop system.

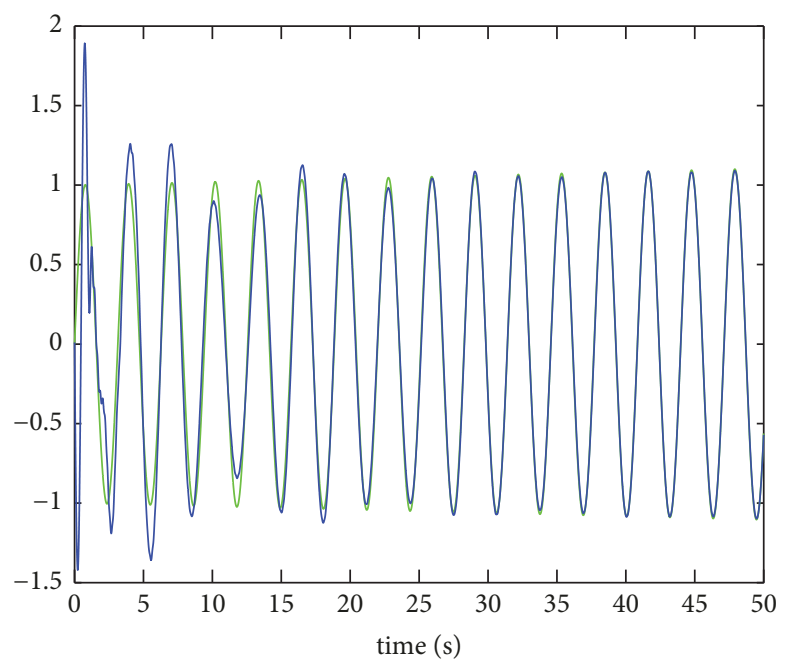

_ Reference signal $F v$
_ Output signal $u(1, t)$

(a) Output tracking

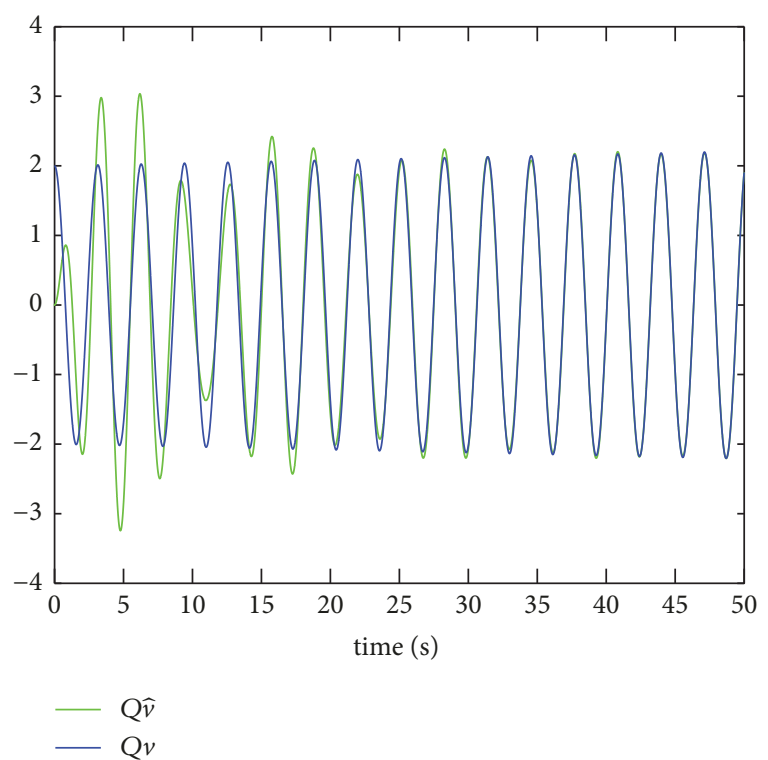

(b) Disturbance estimation

FIGURE 2: Output tracking and disturbance estimation. 
Figure 2(a) shows that the output signal $u(1, t)$ tracks the given reference signal $Y_{r e f}(t)$ as time evolves for noncollocated case. Obviously, output signal $u(1, t)$ can track the given reference signal asymptotically. The disturbance estimation is plotted in Figure 2(b). The observer reconstructs the corresponding disturbance. Both of them show that the convergence is very effective and smooth. More importantly, no peaking phenomenon takes place. Comparing Figure 2(a) with Figure 2(b), it can be seen that after the convergence of the observer, the output converges to zero verifying disturbance rejection.

\section{Concluding Remarks}

In this paper, we mainly solved the performance output regulation problem for a wave-heat cascade system with unmatched disturbance. Applying a novel auxiliary trajectory method and servomechanism design, the performance output can track the given signal asymptotically. Thus, the contribution of this paper is to solve the noncollocated performance output regulation problem of the cascade system despite unmatched disturbance. The idea is potentially promising for treating other PDE-PDE or PDE-ODE systems to solve the performance output regulation which will be our future work.

\section{Data Availability}

The data used to support the findings of this study are available from the corresponding author upon request.

\section{Conflicts of Interest}

The author declares no conflicts of interest.

\section{Acknowledgments}

This work was supported by the National Natural Science Foundation of China (No. 11671240) and the National Natural Science Foundation of China for the Youth (No. 11801339).

\section{References}

[1] W. Guo and B.-Z. Guo, "Performance output tracking for a wave equation subject to unmatched general boundary harmonic disturbance," Automatica, vol. 68, pp. 194-202, 2016.

[2] X. Zhang, H. Feng, and S. Chai, "Performance output exponential tracking for a wave equation with a general boundary disturbance," Systems \& Control Letters, vol. 98, pp. 79-85, 2016.

[3] H. Feng and B.-Z. Guo, "A new active disturbance rejection control to output feedback stabilization for a one-dimensional anti-stable wave equation with disturbance," Institute of Electrical and Electronics Engineers Transactions on Automatic Control, vol. 62, no. 8, pp. 3774-3787, 2017.

[4] H.-C. Zhou and G. Weiss, "Solving the regulator problem for a 1-D Schrödinger equation via backstepping," IFACPapersOnLine, vol. 50, no. 1, pp. 4516-4521, 2017.

[5] J. Liu, J. Wang, and Y. Guo, "Output tracking for onedimensional schrödinger equation subject to boundary disturbance," Asian Journal of Control, vol. 20, no. 2, pp. 659-668, 2018.
[6] J. Deutscher and S. Kerschbaum, "Output regulation for coupled linear parabolic PIDEs,” Automatica, vol. 100, pp. 360-370, 2019.

[7] F.-F. Jin and B.-Z. Guo, "Performance boundary output tracking for one-dimensional heat equation with boundary unmatched disturbance," Automatica, vol. 96, pp. 1-10, 2018.

[8] H.-C. Zhou and B.-Z. Guo, "Performance output tracking for one-dimensional wave equation subject to unmatched general disturbance and non-collocated control," European Journal of Control, vol. 39, pp. 39-52, 2018.

[9] W. Guo, H.-C. Zhou, and M. Krstic, "Adaptive error feedback regulation problem for $1 \mathrm{D}$ wave equation," International Journal of Robust and Nonlinear Control, vol. 28, no. 15, pp. 4309-4329, 2018.

[10] L. Paunonen and S. Pohjolainen, "Internal model theory for distributed parameter systems," SIAM Journal on Control and Optimization, vol. 48, no. 7, pp. 4753-4775, 2010.

[11] L. Paunonen and S. Pohjolainen, "Robust controller design for infinite-dimensional exosystems," International Journal of Robust and Nonlinear Control, vol. 24, no. 5, pp. 825-858, 2014.

[12] M. Krstic, "Compensating a string PDE in the actuation or sensing path of an unstable ODE," IEEE Transactions on Automatic Control, vol. 54, no. 6, pp. 1362-1368, 2009.

[13] S. Tang and C. Xie, "Stabilization for a coupled PDE-ODE control system," Journal of The Franklin Institute, vol. 348, no. 8, pp. 2142-2155, 2011.

[14] B.-Z. Guo, J.-J. Liu, A. S. Al-Fhaid, A. M. M. Younas, and A. Asiri, "The active disturbance rejection control approach to stabilisation of coupled heat and ODE system subject to boundary control matched disturbance," International Journal of Control, vol. 88, no. 8, pp. 1554-1564, 2015.

[15] H.-C. Zhou, B.-Z. Guo, and Z.-H. Wu, "Output feedback stabilisation for a cascaded wave PDE-ODE system subject to boundary control matched disturbance," International Journal of Control, vol. 89, no. 12, pp. 2396-2405, 2016.

[16] V. Natarajan, D. S. Gilliam, and G. Weiss, "The state feedback regulator problem for regular linear systems," IEEE Transactions on Automatic Control, vol. 59, no. 10, pp. 2708-2723, 2014.

[17] L. Paunonen and S. Pohjolainen, "The internal model principle for systems with unbounded control and observation," SIAM Journal on Control and Optimization, vol. 52, no. 6, pp. 39674000, 2014

[18] H. Feng and X. H. Wu, "Trajectory Planning Approach to Output Regulation for a 1-D Heat Equation with Two Noncollocated Configurations," Automatica, In press.

[19] M. Krstic and A. Smyshlyaev, Boundary Control of PDEs: A Course on Backstepping Designs, SIAM Advances in Design and Control, SIAM, Philadelphia, Pa, USA, 2008. 


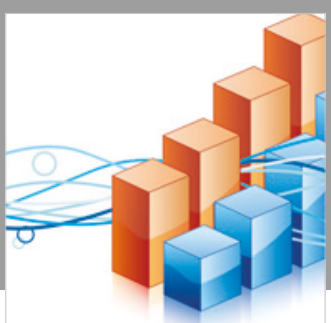

Advances in

Operations Research

\section{-n-m}
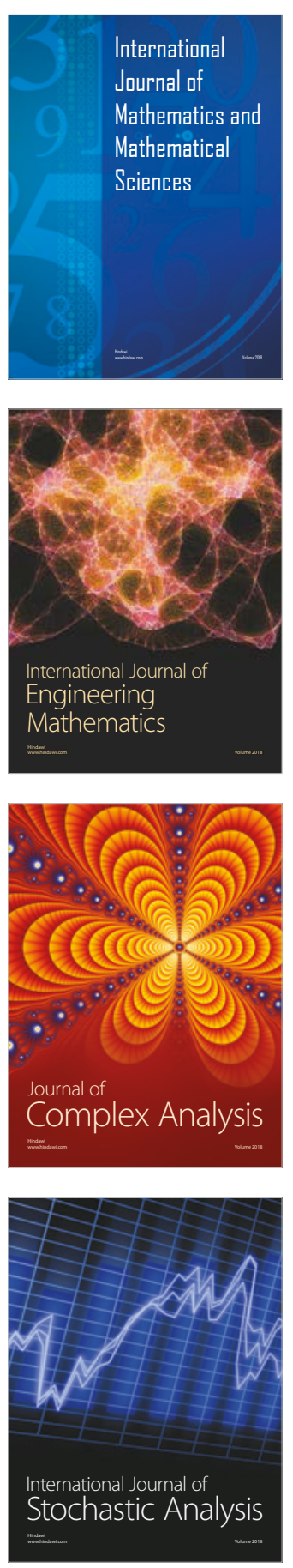
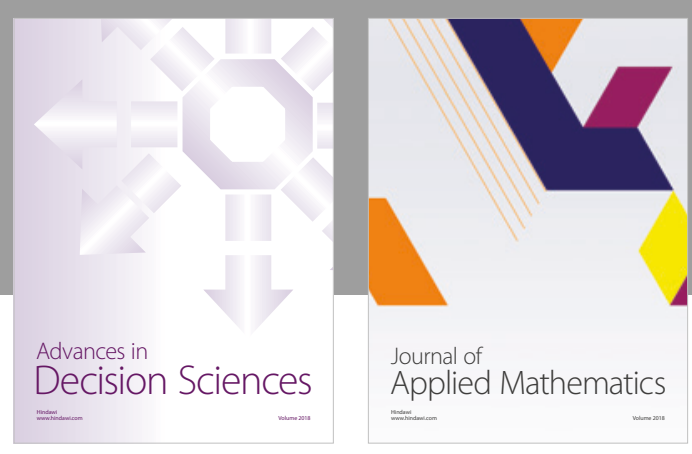

Journal of

Applied Mathematics
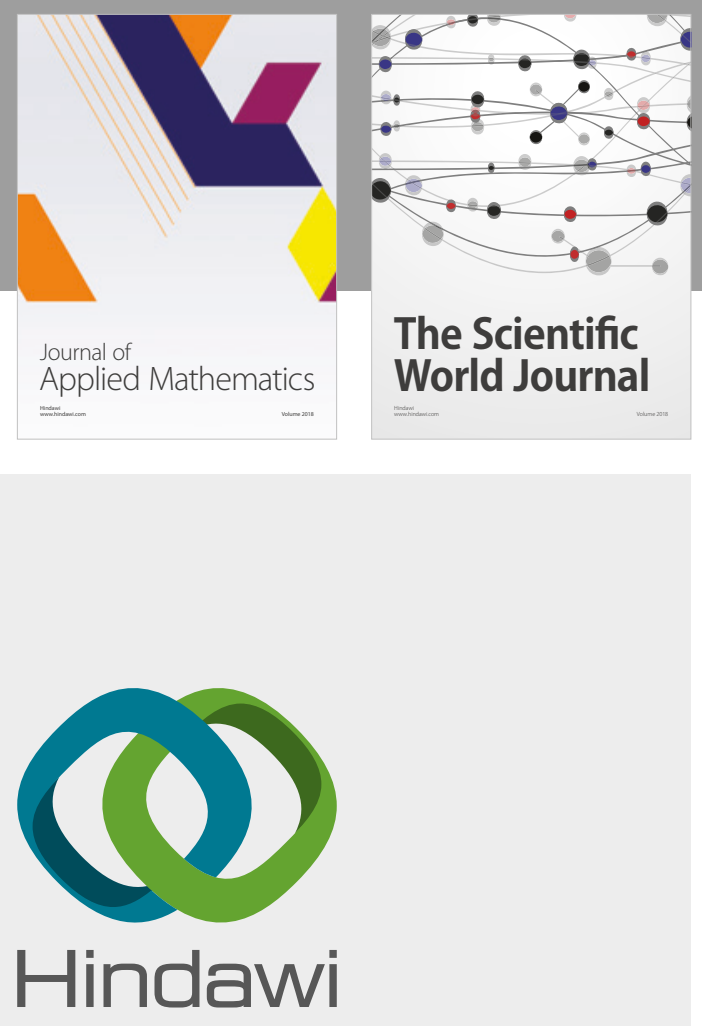

Submit your manuscripts at

www.hindawi.com

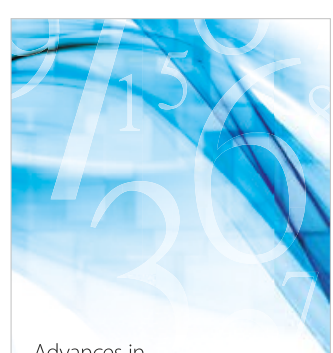

Advances in
Numerical Analysis
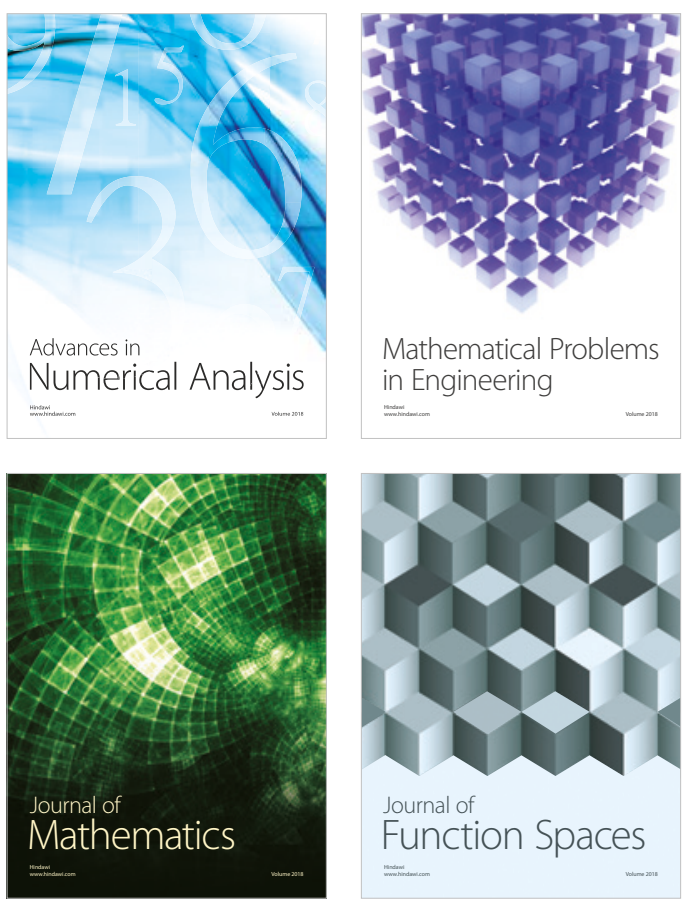

Mathematical Problems in Engineering

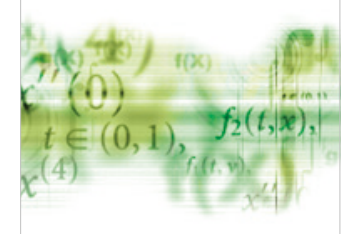

International Journal of

Differential Equations

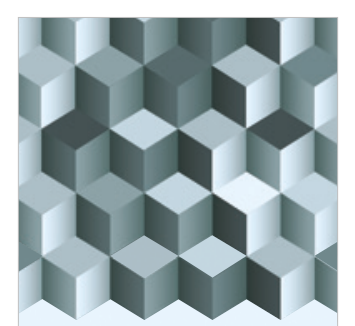

Journal of

Function Spaces
The Scientific

World Journal

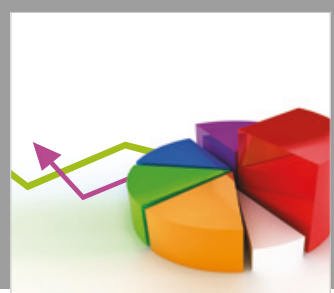

Journal of

Probability and Statistics
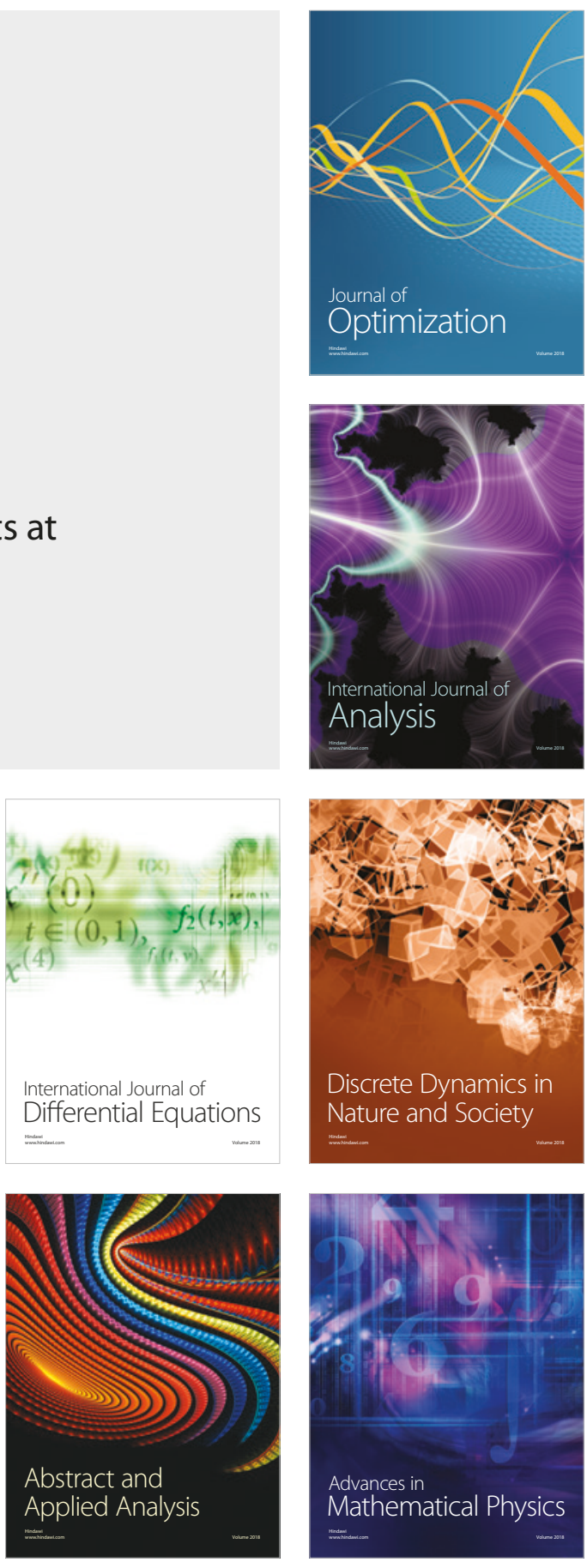